\title{
Competitive Paging Algorithms
}

\author{
Amos Fiat, ${ }^{*, 1}$ Richard M. Karp, ${ }^{\dagger, 2}$ Michael Luby, ${ }^{\ddagger, 3}$ \\ Lyle A. McGeoch, ${ }^{\S}$ Daniel D. Sleator, ${ }^{\prime, 4}$ and Neal E. Young ${ }^{\#, 5}$
}

${ }^{*}$ Department of Computer Science, Tel Aviv University, Tel Aviv, Israel 69978;

${ }^{\dagger}$ Department of Electrical Engineering and Computer Science, Computer Science Division, University of California, Berkeley, CA 94720;

${ }^{\ddagger}$ Department of Computer Science, University of Toronto, Toronto, Ontario, Canada M6C 3B7;

${ }^{\S}$ Department of Mathematics and Computer Science, Amherst College, Amherst, Massachusetts 01002;

"School of Computer Science, Carnegie Mellon University, Pittsburgh, Pennsylvania 15213; and

${ }^{*}$ Computer Science Department, Princeton University, Princeton, New Jersey 08544.

Received November 1988; revised October 1990

The paging problem is that of deciding which pages to keep in a memory of $k$ pages in order to minimize the number of page faults. We develop the marking algorithm, a randomized on-line algorithm for the paging problem. We prove that its expected cost on any sequence of requests is within a factor of $2 \mathrm{H}_{k}$ of optimum. (Where $H_{k}$ is the $k$ th harmonic number, which is roughly $\ln k$.) The best such factor that can be achieved is $H_{k}$. This is in contrast to deterministic algorithms, which cannot be guaranteed to be within a factor smaller than $k$ of optimum. An alternative to comparing an on-line algorithm with the optimum off-line algorithm is the idea of comparing it to several other on-line algorithms. We have obtained results along these lines for the paging problem. Given a set of on-line algorithms

\footnotetext{
${ }^{1}$ Support was provided by a Weizmann fellowship.

${ }^{2}$ Partial support was provided by the International Computer Science Institute, Berkeley, CA, and by NSF Grant CCR-8411954.

${ }^{3}$ Support was provided by the International Computer Science Institute and operating grant A8092 of the Natural Sciences and Engineering Research Council of Canada. Current address: International Computer Science Institute, Berkeley, CA 94704.

${ }^{4}$ Partial support was provided by DARPA, ARPA order 4976, Amendment 20, monitored by the Air Force Avionics Laboratory under Contract F33615-87-C-1499, and hy the National Science Foundation under Grant CCR-8658139.

${ }^{5}$ Part of this work was performed while the author was at the Digital Equipment Corp. Systems Research Center, Palo Alto, CA.
} 
and a set of appropriate constants, we describe a way of constructing another on-line algorithm whose performance is within the appropriate constant factor of each algorithm in the set. (c) 1991 Academic Press, Inc.

\section{INTRODUCTION}

Consider a memory system with $k$ pages of fast memory (a cache) and $n-k$ pages of slow memory. A sequence of requests to pages is to be satisfied, and in order to satisfy a request to a page that page must be in fast memory. If a requested page is not in fast memory a page fault occurs. In this case the requested page must be moved into fast memory, and (usually) a page must be moved from fast memory to slow memory to make room for the requested page. The paging problem is that of deciding which page to eject from fast memory. The cost to be minimized is the number of page faults.

A paging algorithm is said to be on-line if its decision of which page to eject from fast memory is made without knowledge of future requests. Sleator and Tarjan [15] analyzed on-line paging algorithms by comparing their performance on any sequence of requests to that of the optimum off-line algorithm (that is, one that has knowledge of the entire sequence of requests in advance). They showed that two strategies for paging (ejecting the least recently used page, or LRU, and first-in-first-out, or FIFO) could be worse than the optimum off-line algorithm by a factor of $k$, but not more, and that no on-line algorithm could achieve a factor less than $k$.

Karlin et al. [9] introduced the term competitive to describe an on-line algorithm whose cost is within a constant factor (independent of the request sequence) of the optimum off-line algorithm, and they used the term strongly competitive to describe an algorithm whose cost is within the smallest possible constant factor of optimum. These authors proposed another paging strategy, flush-when-full or FWF, and showed that it is also strongly $k$-competitive.

Manasse et al. [10] extended the definition of competitiveness to include randomized on-line algorithms (on-line algorithms which are allowed to use randomness in deciding what to do). Let $A$ be a randomized on-line algorithm, let $\sigma$ be a sequence of requests, and let $\overline{C_{A}}(\sigma)$ be the cost of algorithm $A$ on sequence $\sigma$ averaged over all the random choices that $A$ makes while processing $\sigma$. Let $C_{B}(\sigma)$ be the cost of deterministic algorithm $B$ on sequence $\sigma$. Algorithm $A$ is said to be $c$-competitive if there is a constant $a$ such that for every request sequence $\sigma$ and every algorithm $B$ :

$$
\overline{C_{A}}(\sigma) \leq c \cdot C_{B}(\sigma)+a
$$


The constant $c$ is known as the competitive factor. This definition has the desirable feature of ensuring that $A$ 's average performance on every individual sequence is close to that of the optimum off-line algorithm.

In this paper we consider randomized algorithms for the paging problem from the competitive point of view. We describe a randomized algorithm, called the marking algorithm, and show that it is $2 \mathrm{H}_{k}$-competitive. (Here $H_{k}$ denotes that $k$ th harmonic number: $H_{k}=1+\frac{1}{2}+$ $\frac{1}{3}+\cdots+1 / k$. This function is closely approximated by the natural logarithms: $\ln (k+1) \leq H_{k} \leq \ln (k)+1$. We also show that no randomized paging algorithm can have a competitive factor less than $H_{k}$.

The marking algorithm is strongly competitive (its competitive factor is $H_{k}$ ) if $k=n-1$, but it is not strongly competitive if $k<n-1$. We describe another algorithm, EATR, which is strongly competitive for the case $k=2$.

Borodin, Linial, and Saks [3] gave the first specific problem in which the competitive factor is reduced if the on-line algorithm is allowed to use randomness. The problem they analyzed is the uniform task system. They presented a randomized algorithm for uniform task systems whose competitive factor is $2 H_{n}$, where $n$ is the number of states in the task system, and proved that for this problem the competitive factor of any randomized algorithm is at least $H_{n}$. The marking algorithm is an adaptation of the randomized algorithm of Borodin $e t a l$. It was discovered by three groups working independently. These three groups collaborated in the writing of this paper.

The standard definition of competitiveness requires that the on-line algorithm be within a constant factor of any other algorithm, even an off-line one. In the case of deterministic paging algorithms this constraint is so severe that the best possible constant required is rather large. An alternative approach is to require that the on-line algorithm be efficient compared to several other on-line algorithms simultaneously. Given deterministic on-line algorithms for the paging problem $B(1), B(2), \ldots, B(m)$, and constants $c(1), c(2), \ldots, c(m)$, we show how to construct a new on-line algorithm whose performance is within a factor of $c(i)$ of $B(i)$ for all $1 \leq i \leq m$, under the condition that $1 / c(1)+\cdots+1 / c(m) \leq 1$. For example, we can construct an algorithm whose performance is within a factor of two of the performance of both the LRU algorithm and the FIFO algorithm. We also show how this construction can be applied to randomized algorithms.

This paper is organized as follows. Section 2 defines server problems (a generalized form of the paging problem) and introduces the terminology we shall use for the paging problem. Section 3 discusses the marking algorithm, Section 4 describes algorithm EATR, Section 5 proves the $H_{k}$ lower bound on the competitive factor, and Section 6 contains our results 
about combining algorithms. Recent extensions to this work are described in Section 7, along with several open problems.

\section{Server Problems}

To put our work on paging in context it is useful to point out the connection between the paging problem and the $k$-server problem. Let $G$ be an $n$-vertex graph with positive edge lengths obeying the triangle inequality, and let $k$ mobile servers occupy vertices of $G$. Given a sequence of requests, each of which specifies a vertex that requires service, the $k$-server problem is to decide how to move the servers in response to each request. If a requested vertex is unoccupied, then some server must be moved there. The requests must be satisfied in the order of their occurrence in the request sequence. The cost of handling a sequence of requests is equal to the total distance moved by the servers.

Server problems were introduced by Manasse, McGeoch, and Sleator $[10,11]$. They showed that no deterministic algorithm for the $k$-server problem can be better than $k$-competitive, they gave $k$-competitive algorithms for the case when $k=2$ and $k=n-1$, and they conjectured that there exists a $k$-competitive $k$-server algorithm for any graph. This conjecture holds when the graph is uniform [15], a weighted cache system (where the cost of moving to a vertex from anywhere is the same) [4], a line [4], or a tree [5]. Fiat et al. [7] showed that there is an algorithm for the $k$-server problem with a competitive factor that depends only on $k$. There has also been work on memoryless randomized algorithms for server problems $[1,6,14]$. These algorithms keep no information between requests except the server locations. The randomized algorithm of Coppersmith et al. [6] is $k$-competitive for a large class of graphs.

In the uniform $k$-server problem the cost of moving a server from any vertex to any other is one. The paging problem is isomorphic to the uniform $k$-server problem. The correspondence between the two problems is as follows: the pages of address space correspond to the $n$ vertices of the graph, and the pages in fast memory correspond to those vertices occupied by servers. In the remainder of this paper we shall use the terminology of the uniform $k$-server problem.

\section{The Marking Algorithm}

The marking algorithm is a randomized algorithm for the uniform $k$-server problem on a graph with $n$ vertices. The algorithm works as follows: The servers are initially on vertices $1,2,3, \ldots, k$. The algorithm 
maintains a set of marked vertices. Initially the marked vertices are exactly those that are covered by servers. After each request, the marks are updated, then a server is moved if necessary, as follows:

Marking. Each time a vertex is requested, that vertex is marked. The moment $k+1$ vertices are marked, all the marks except the one on the most recently requested vertex are erased.

Serving. If the requested vertcx is alrcady covered by a server, then no servers move. If the requested vertex is not covered, then a server is chosen uniformly at random from among the unmarked vertices, and this server is moved to cover the requested vertex.

This algorithm can be interpreted as a randomized form of LRU as follows. Rather than maintaining one queue of servers, the algorithm maintains two of them. When a server is needed it is taken from the front of one of the queues and placed at the end of the other. When the queue from which servers are taken is empty it is replaced by the other queue, but not before the order of the elements in the queue is shuffled by a random permutation.

THEOREM 1. The marking algorithm is a $2 H_{k}$-competitive algorithm for the uniform $k$-server problem on $n$ vertices.

Proof. Let $\sigma=\sigma(1), \sigma(2), \ldots$ be a sequence of requests. The marking algorithm (denoted $M$ ) implicitly divides $\sigma$ (excluding some requests at the beginning) into phases. The first phase begins with $\sigma(i)$, where $i$ is the smallest integer such that $\sigma(i) \notin\{1,2, \ldots, k\}$. In general the phase starting with $\sigma(i)$ ends with $\sigma(j)$, where $j$ is the smallest integer such that the set $\{\sigma(i), \sigma(i+1), \ldots, \sigma(j+1)\}$ is of cardinality $k+1$.

At the start of every phase, the marked vertices are precisely the ones occupied by $M$ 's servers. The first request of every phase is to an unmarked vertex. A vertex is called clean if it was not requested in the previous phase and has not yet been requested in this phase. A vertex is called stale if it was requested in the previous phase but has not yet been requested in this phase.

Our proof is organized as follows. We let an adversary choose any algorithm $A$. We then evaluate the cost incurred by $A$ during a phase, evaluate the cost incurred by $M$ during the same phase, and compare these two quantities. These costs depend on $l$, the number of requests to clean vertices during the phase.

Without loss of generality, we shall assume that the algorithm $A$ chosen by the adversary is lazy. A lazy algorithm is one which does not move any server in response to a request to a covered vertex and moves exactly one server in response to a request to an uncovered vertex. Manasse et al. 
$[10,11]$ showed that for any given algorithm, there is always a lazy one that incurs no more cost. Thus our assumption does not limit the generality of our result.

We shall first argue that the amortized cost incurred by $A$ over the phase is at least $l / 2$. Let $d$ be the number of $A$ 's servers that do not coincide with any of $M$ 's servers at the beginning of the phase. Let $d^{\prime}$ be this quantity at the end of the phase. Let $C_{A}$ be the cost incurred by $A$ in the phase. We claim $C_{A} \geq l-d$, because among the $l$ requests to clean vertices at most $d$ of these will be for vertices that $A$ already covers.

A second bound on $C_{A}$ is obtained by considering $S$, the set of marked vertices at the end of the phase. The vertices of $S$ are those that are covered by $M$ at the end of the phase, so at the end of the phase $d^{\prime}$ servers of $A$ are not in $S$. During this phase exactly the vertices of $S$ were requested, so since $A$ is lazy, we know that at least $d^{\prime \prime}$ of $A$ 's servers were outside of $S$ during the entire phase. The remaining $k-d^{\prime}$ servers had to cover requests at each of the $k$ vertices of $S$, implying that $A$ 's cost is at least $d^{\prime}$. This is, $C_{A} \geq d^{\prime}$.

Combining the inequalities from the preceding paragraphs we obtain

$$
C_{A} \geq \max \left(l-d, d^{\prime}\right) \geq \frac{1}{2}\left(l-d+d^{\prime}\right) .
$$

When this is summed over all phases, the $d$ and $d^{\prime}$ terms telescope, so we can assume for the purposes of this analysis that the cost of a phase is just $l / 2$.

We shall now bound the expected cost incurred by $M$ during the phase. There are $l$ requests to clean vertices and each of these costs one. There are $k-l$ requests to stale vertices; the expected cost of each of these requests is just the probability that there is no server there. This probability varies as a function of the current number of stale vertices, $s$, and the number of clean vertices requested in the phase so far, $c$. The expected cost of the request is $c / s$ because there are $c$ unserved vertices distributed uniformly among $s$ stale vertices.

During the phase, the sequence will make $l$ requests to clean vertices and $k-l$ requests to stale vertices. The sequence with the highest expected cost for $M$ is the one which first requests all the clean vertices (increasing $c$ ), before requesting any stale vertices. The expected cost of the requests to stale vertices is thus bounded by

$$
\frac{l}{k}+\frac{l}{k-1}+\frac{l}{k-2} \cdots+\frac{l}{l+1}=l\left(H_{k}-H_{l}\right) .
$$


The total expected cost to $M$ for the phase is therefore at most

$$
l\left(H_{k}-H_{l}+1\right) \leq l H_{k} .
$$

Since the cost incurred by $A$ during the phase is (amortized) $l / 2$, this proves that the marking algorithm is $2 H_{k}$-competitive.

In the special case of $n-1$ servers, we can obtain a tighter bound.

THEOREM 2. The marking algorithm is a $H_{n-1}$-competitive algorithm for the uniform $(n-1)$-server problem on $n$ vertices.

Proof. The above proof can be modified slightly to give this theorem. In the $(n-1)$-server problem, every phase has $l=1$. In this case we can show that the amortized cost of $A$ is at least $l$ per phase.

As above, let $d$ be the number of $A$ 's servers that do not coincide with any of $M$ 's servers at the beginning of the phase and $d^{\prime}$ be this quantity at the end of the phase. The first request of the phase is to a clean vertex, and its cost to $A$ is at least $1-d$. Among the $n-2$ other vertices requested in this phase, at least $d^{\prime}$ cause $A$ to incur a cost of one. Thus the cost to $A$ is at least $1-d+d^{\prime}$. This shows that the amortized cost to $A$ of a phase is at least 1 . Combining this with the preceding analysis of $M$ finishes the proof.

Why is it that if $l=1$ we can show that the cost to $A$ is at least $l-d+d^{\prime}$, but when $l>1$ we can only show that the cost is at least $\left(l-d+d^{\prime}\right) / 2$ ? The distinction is due to a difference in the structure of the requests in a phase. The tighter bound actually holds whenever the phase has the following structure: after the last request to a clean vertex, all of the other $k-1$ vertices used during the phase are requested. In this case $A$ incurs a cost of $l-d$ for the clean vertices, then an additional cost of $d^{\prime}$ for the subsequent requests to the other $k-1$ vertices. This pattern holds for the case $l=1$.

The marking algorithm is not in general $H_{k}$-competitive for the uniform $k$-server problem. This is even true in the case $k=2, n=4$. Suppose that the servers of $M$ are initially on vertices 1 and 2, and the servers of the adversary $A$ are on vertices 1 and 3 . The first phase will consist of requests to vertices 3 and 4 . The marking algorithm will incur a cost of 2 for these requests, and end with servers on vertices 3 and 4 . To handle this phase, algorithm $A$ will use its server on vertex 3 to cover the request on vertex 4 , incurring a cost of 1 . At the end of the phase the servers of $A$ and $M$ will again coincide on exactly one vertex, and the process can be repeated. The competitive factor for this application of $M$ is 2, which exceeds $H_{2}$. 


\section{Algorithm EATR}

Algorithm EATR is a randomized algorithm for the uniform 2-server problem. (The name stands for "end after twice requested," a rough description of how the algorithm defines the end of a phase.)

The servers are initially located on vertices 1 and 2 . The algorithm partitions the sequence of requests into phases in a way that is different from that used by the marking algorithm. The first phase starts at the first request that is to neither 1 nor 2 . A vertex is called clean if it was not occupied by a server at the end of the previous phase and has not been requested during this phase. A vertex is called stale if it is not clean and is not the most recently requested vertex. The algorithm maintains one server on the most recently requested vertex, and the other uniformly at random among the set of stale vertices. When a stale vertex is requested the servers are placed on the two most recently requested vertices. The next phase begins after this, on a request for a vertex that is not covered by a server.

THEOREM 3. Algorithm EATR is a 3/2-competitive algorithm for the uniform 2-server problem.

Proof. Let $l$ be the number of clean vertices requested during a phase. Before the request to the stale vertex that terminates the phase, the number of stale vertices is $l+1$, and there is a server on each of these with probability $1 /(l+1)$. The expected cost of a phase to EATR is then

$$
l+\frac{l}{l+1} .
$$

The phases as defined by EATR have the special structure described in the paragraph after the proof of Theorem 2 . Thus the amortized cost incurred by any algorithm for a phase is at least $l$. The competitive factor is therefore at most

$$
\frac{l+l /(l+1)}{l}=1+\frac{1}{(l+1)} \leq \frac{3}{2} .
$$

\section{A LOWER BOUND}

THEOREM 4. There is no c-competitive randomized algorithm for the uniform $(n-1)$-server problem on $n$ vertices with $c<H_{n-1}$. 
Proof. ${ }^{1}$ Let $A$ be a randomized on-line algorithm for solving the problem. We use the technique of constructing a nemesis sequence for algorithm $A$. Since $A$ is randomized, the adversary constructing the sequence is not allowed to see where the servers are. The adversary is, however, able to maintain a vector $p=\left(p_{1}, p_{2}, \ldots, p_{n}\right)$ of probabilities, where $p_{i}$ is the probability that vertex $i$ is not covered by a server. (The adversary can do this by simulating $A$ on all possible outcomes of its random choices and condensing the information about where the servers are in each of these simulations into the vector of probabilities.) Note that $\sum_{i} p_{i}=1$.

If the nemesis sequence requests a vertex $i$, then the expected cost incurred by $A$ is $p_{i}$. As a result of responding to the request, $p_{i}$ changes to 1 , and some other elements of the probability vector may decrease. (Even if we allow $A$ to change the vector arbitrarily and ignore the cost it incurs in doing so, the lower bound still holds.)

The adversary will maintain a set of marked vertices for the sequence it has generated so far in just the way that the marking algorithm would. Furthermore, we can also define phases in the nemesis sequence just as we did for an arbitrary sequence processed by the marking algorithm. As usual, at the start of each phase $n-1$ vertices are marked. After the first request of the phase, one vertex is marked.

Armed with these tools (the marking and the probability vector), the adversary can generate a sequence such that the expected cost of each phase to $A$ is $H_{n-1}$, and the cost to the optimum off-line algorithm is 1 . This will prove the theorem.

Consider a situation in which the number of unmarked vertices is $u$. The goal of the adversary is to generate some requests that cause $A$ to incur an expected cost of at least $1 / u$ and decrease the number of unmarked vertices to $u-1$ (except if $u=1$, in which case the number of unmarked vertices changes to $n-1)$. Since $u$ takes on every integer value between 1 and $n-1$ the total expected cost incurred by $A$ is at least $H_{n-1}$. This subsequence of requests will be called a subphase. Constructing a subphase will show how to generate the desired nemesis sequence and complete the proof of the theorem.

A subphase consists of zero or more requests to marked vertices, followed by a request to an unmarked vertex. Let $S$ be the set of marked vertices, and let $P=\Sigma_{i \in S} p_{i}$. Let $u$ be $n-|S|$, the number of unmarked vertices. If $P=0$ then there must be an unmarked vertex $i$ with $p_{i} \geq 1 / u$. In this case the subphase consists of a single request to $i$. The expected cost of this request is at least $1 / u$.

\footnotetext{
${ }^{1}$ Raghavan [13, pp. 118-119] presents a different proof of this theorem based on a generalization of the minimax principle due to Andy Yao [16].
} 
If $P>0$, then there must be $i \in S$ such that $p_{i}>0$. Let $\varepsilon=p_{i}$, and let the first request of the subphase be $i$. Next, a set of requests are generated by the following loop ( $P$ denotes the current total probability of the marked vertices):

While $P>\varepsilon$ and while the total expected cost of all the requests in this subphase so far does not exceed $1 / u$, request vertex $i \in S$, where $p_{i}=\max _{j \in S}\left(p_{j}\right)$.

Each iteration of this loop adds at least $\varepsilon /|S|>0$ to the total expected cost of this subphase. Thus the loop must terminate. If the total cxpected cost ends up exceeding $1 / u$, then an arbitrary request is made to an unmarked vertex, and the subphase is over. If the loop terminates with $P \leq \varepsilon$, then a request is generated to the unmarked vertex $j$ with the highest probability value. Note that $p_{j} \geq(1-P) / u$. The following inequalities finish the proof:

expected cost of the subphase

$$
\geq \varepsilon+p_{j} \geq \varepsilon+\frac{1-P}{u} \geq \varepsilon+\frac{1-\varepsilon}{u} \geq \frac{1}{u} .
$$

If there are $k$ servers, with $1 \leq k \leq n-1$, then the adversary can ignore all but $k+1$ vertices of the graph and force the on-line algorithm to incur a cost at least $H_{k}$ times optimum. Thus we have:

COROllary 5. There is no c-competitive randomized algorithm for the uniform $k$-server problem on a graph of $n$ vertices with $c<H_{k}$, where $1 \leq k \leq n-1$.

\section{Algorithms That Are Competitive Against SeVERAL Others}

In many applications of the $k$-server model, the following situation arises: one is given several on-line algorithms with desirable characteristics and would like to construct a single on-line algorithm that has the advantages of all the given ones. For example, in the case of the paging problem (the uniform-cost $k$-server problem), the least-recently-used page replacement algorithm (LRU) is believed to work well in practice, but, in the worst case, it can be $k$ times as costly as the optimal off-line algorithm. On the other hand, we have exhibited a randomized on-line algorithm that is $2 \mathrm{H}_{k}$-competitive, and thus it has theoretical advantages over LRU. Can we construct an on-line algorithm that combines the advantages of these two algorithms? We shall see that the answer is "Yes." 
We adopt the viewpoint that each on-line algorithm is tailored for a particular choice of $k$, the number of servers, and $n$, the number of vertices that may request service. We may assume without loss of generality that the $n$ vertices are named by the integers $1,2, \ldots, n$. The ordered pair $(k, n)$ is called the type of the algorithm. Thus, in a request sequence presented to an algorithm of type $(k, n)$, each request is an integer between 1 and $n$. According to this viewpoint a general strategy (such as LRU, FIFO, or the marking algorithm) determines infinitely many individual algorithms, corresponding to all the possible choices of $k$ and $n$.

Let $A$ and $B$ be deterministic on-line algorithms of the same type. Let $c$ be a positive constant. Then $A$ is said to be $c$-competitive against $B$ if there exists a constant $a$ such that on every sequence $\sigma$ of requests,

$$
C_{A}(\sigma) \leq c \cdot C_{B}(\sigma)+a .
$$

Let $c^{*}=(c(1), c(2), \ldots, c(m))$ be a sequence of positive real numbers. Then $c^{*}$ is said to be realizable if, for every type $(k, n)$, and for every sequence $B(1), B(2), \ldots, B(m)$ of deterministic on-line algorithms of type $(k, n)$, there exists a deterministic on-line algorithm $A$ of type $(k, n)$ such that, for $i=1,2, \ldots, m, A$ is $c(i)$-competitive against $B(i)$.

THEOREM 6. The sequence $c^{*}$ is realizable if and only if

$$
\sum_{1 \leq i \leq m} \frac{1}{c(i)} \leq 1 .
$$

Proof. (Sufficiency) We show that, if (1) holds, then $c^{*}$ is realizable. Let deterministic on-line algorithms $B(1), B(2), \ldots, B(m)$ of type $(k, n)$ be given. We shall construct a deterministic on-line algorithm $A$ of type $(k, n)$ such that, for all positive integers $r$, all request sequences $\sigma$, and all $i$ between 1 and $m, B(i)$ incurs a cost greater than or equal to $[r / c(i)]$ by the time $A$ incurs cost $r$. This will prove the sufficiency of (1).

Algorithm $A$ will be a lazy algorithm; i.e., it will move a server only when a vertex is requested that is currently not covered by a server. Let $\sigma$ be a fixed request sequence. The time interval $\left(t_{1}, t_{2}\right)$ is called a $v$-interval for $A$ if, when processing $\sigma, A$ moves a server to vertex $v$ at time $t_{1}$, leaves the server on vertex $v$ until time $t_{2}$, and then moves that server at time $t_{2}$. Algorithm $A$ is said to punish algorithm $B$ at time $t_{2}$ if, for some $v$ and $t_{1},\left(t_{1}, t_{2}\right)$ is a $v$-interval for $A$, and, for some $\left(t_{1}^{\prime}, t_{2}^{\prime}\right)$ such that $t_{1}^{\prime} \leq t_{1}<t_{2}^{\prime} \leq t_{2},\left(t_{1}^{\prime}, t_{2}^{\prime}\right)$ is a $v$-interval for $B$. Clearly, $C_{B}(\sigma)$ is at least as great as the number of time steps at which $A$ punishes $B$. Thus, it suffices to show that $A$ can punish each $B(i)$ at least $\left[C_{A}(\sigma) / c(i)\right]$ times. 
We shall show that, at each step at which $A$ incurs a unit of cost, $A$ has complete freedom to decide which algorithm $B(i)$ to punish. Let $S(A, t)$ be the set of vertices that $A$ covers by servers just before request $\sigma(t)$ arrives and suppose that $\sigma(t) \notin S(A, t)$, so that $A$ must incur a unit of cost in order to process $\sigma(t)$. Let $S(B(i), t+1)$ be the set of vertices that $B(i)$ covers by servers just after processing $\sigma(t)$. Since the sets $S(A, t)$ and $S(B(i), t+1)$ each have cardinality $k$, and since $S(B(i), t+1)$ contains $\sigma(t)$ but $S(A, t)$ does not, there must be some vertex $u$ that lies in $S(A, t)$ but does not lie in $S(B(i), t+1)$. Then $A$ can punish $B(i)$ at step $t$ by moving a server from vertex $u$ to vertex $\sigma(t)$.

Let $\operatorname{PUN}(i, s, \sigma)$ denote the number of times $A$ punishes $B(i)$ while processing the first $s$ requests in $\sigma$, and let $C_{A}(s, \sigma)$ denote the cost that $A$ incurs during the processing of the first $s$ elements of $\sigma$. Suppose that $A$ must move a server in order to process $\sigma(t)$. Then it chooses the server to move in such a way as to punish that algorithm $B(i)$ for which $c(i)(\operatorname{PUN}(i, t-1, \sigma)+1)$ is least. It is easily verified that, provided $\sum 1 / c(i) \leq 1$, the following holds for all positive integers $r$ and all $i: B(i)$ gets punished at least $\lfloor r / c(i)\rfloor$ times by the time $A$ incurs a cost of $r$. This completes the proof of the sufficiency of (1).

(Necessity) Let $m$ be a positive integer. Let $c^{*}=(c(1), c(2), \ldots, c(m))$ be such that $\Sigma 1 / c(i)>1$. We construct on-line deterministic algorithms $B(1), B(2), \ldots, B(m)$ of type $(2 m-1,2 m)$ such that no on-line deterministic algorithm $A$ can be $c(i)$-competitive against each $B(i)$. Since $n=2 m$ and $k=2 m-1$, it will be the case that, at any step in the execution of an on-line deterministic algorithm, exactly one of the $2 m$ possible vertices fails to be covered by a server. For $i=1,2, \ldots, m$ let $B(i)$ be the algorithm that keeps all vertices except $i$ and $i+m$ permanently covered by servers and that shuttles the remaining server between $i$ and $i+m$ in response to requests for those two vertices. No two of these algorithms are ever required to move a server at the same time. At any stage in the execution of a deterministic on-line algorithm $A$ there will exist some vertex that is not covered; this is true because there are $2 m$ vertices and only $2 m-1$ servers. Thus, given any deterministic on-line algorithm $A$ and any positive integer $N$, it is possible to construct a request sequence $\tau(N)$ of length $N$ that causes $A$ to move a server at every step. Then $C_{A}(\tau(N))=N$, and $\sum C_{B(i)}(\tau(N)) \leq N$. If $A$ is to be $c(i)$-competitive with each of the on-line algorithms $B(i)$ then there must exist constants $a(i)$ such that, for all $i$ and all $N$,

$$
C_{A}(\sigma(N)) \leq c(i) \cdot C_{B(i)}(\sigma(N))+a(i) .
$$

But these inequalities, together with the fact that $\Sigma 1 / c(i)>1$, lead to a contradiction for sufficiently large $N$. 
We now extend our definitions to the case of randomized algorithms. Let $A$ and $B$ be randomized on-line algorithms of the same type. Let us say that algorithm $A$ is c-competitive against algorithm $B$ if there exists a constant $a$ such that, for every request sequence $\sigma$,

$$
\overline{C_{A}}(\sigma) \leq c \cdot \overline{C_{B}}(\sigma)+a .
$$

Let $c^{*}=(c(1), c(2), \ldots, c(m))$ be a sequence of positive reals. Then $c^{*}$ is said to be $r$-realizable if for every type $(k, n)$, and for every sequence $B(1), B(2), \ldots, B(m)$ of randomized on-line algorithms of type $(k, n)$, there exists a randomized on-line algorithm $A$ of type $(k, n)$ such that, for $i=1,2, \ldots, m, A$ is $c(i)$-competitive against $B(i)$.

Theorem 7. If $c^{*}$ is realizable then $c^{*}$ is r-realizable.

Proof. Our proof is modeled after the proof of sufficiency in Theorem 6. In that proof, deterministic on-line algorithms $B(1), B(2), \ldots, B(m)$ of type $(k, n)$ were given, and the deterministic on-line algorithm $A$ of type $(k, n)$ was constructed to be $c(i)$-competitive against $B(i)$ for each $i$. The construction had the property that the action of $A$ in response to the $t$ th request in an input sequence $\sigma$ was completely determined by $c^{*}$ and the actions of the algorithms $B(i)$ in response to the first $t$ requests in $\sigma$. The construction ensures that, if $c^{*}$ is realizable, then $B(i)$ incurs cost at least $\lfloor r / c(i)\rfloor$ by the time $A$ incurs cost $r$, and hence $C_{B(i)}(\sigma) \geq\left\lfloor C_{A}(\sigma) / c(i)\right\rfloor$. Let us call this construction PUNISH.

We shall extend PUNISH to the randomized case in a straightforward manner. A randomized on-line algorithm may be viewed as basing its actions on the request sequence $\sigma$ presented to it and on an infinite sequence $\rho$ of independent unbiased random bits. The action of the algorithm on $\sigma(t)$, the $t$ th request in $\sigma$, will be determined by the first $t$ requests in $\sigma$ and by some initial part of the infinite sequence $\rho$. Let $C_{B}(\sigma, \rho)$ be the cost incurred when algorithm $B$ is executed on request sequence $\sigma$ using the sequence $\rho$ of random bits. Then $\overline{C_{B}}(\sigma)$ is the expected value of $C_{B}(\sigma, \rho)$.

Let $c^{*}$ be a realizable sequence, and let $B(1), B(2), \ldots, B(m)$ be randomized on-line algorithms. We shall construct a randomized on-line algorithm $A$ of the same type that is $c(i)$-competitive against $B(i)$, for $i=1,2, \ldots, m$. We begin by giving a conceptual view of algorithm $A$, ignoring questions of effectiveness. Algorithm $A$ starts by constructing an infinite sequence $\rho$ of independent, unbiased bits. Then, as successive requests in the input sequence $\sigma$ arrive, it calculates the actions of each of the $B(i)$ on these requests in $\sigma$ using the sequence of random bits $\rho$, and determines its own actions by applying PUNISH to $c^{*}$ and the actions of $B(1), B(2), \ldots, B(m)$ on $\sigma$ with random bits $\rho$. This ensured that, for 
$i=1,2, \ldots, m, C_{B(i)}(\sigma, \rho) \geq\left\lfloor C_{A}(\sigma, \rho) / c(i)\right\rfloor$, and, averaging over all choices of the random bits $\rho, \overline{C_{A}}(\sigma) \leq c(i) \cdot \overline{C_{B}}(\sigma)+a(i)$.

To do this simulation it is not actually necessary for $A$ to generate an infinite sequence $\rho$. To process requests $1,2, \ldots, t, A$ needs to generate as many random bits as are required by any $B(i)$. Algorithm $A$ must also remember that portion of the sequence $\rho$ that it has given to some $B(i)$, but not all of them. Of course, $A$ is also required to simulate the behavior of each $B(i)$ on the given input sequence.

\section{EXTENSIONS}

The problem of devising a strongly competitive algorithm for any $k$ and $n$ was solved by McGeoch and Sleator [12]. Their partitioning algorithm is much more complicated than the marking algorithm, but achieves the optimal competitive factor of $H_{k}$.

For deterministic server problems all evidence indicates that the optimal competitive factor is $k$, and is therefore independent of the distances in the graph $[4,5,11]$. This is not true in the randomized case. Karlin et al. [8] have shown that for two servers in a graph that is an isosceles triangle the best competitive factor that can be achieved is a constant that approaches $e /(e-1) \cong 1.582$ as the length of the similar sides go to infinity. This contrasts with the uniform 3-vertex, 2-server problem for which the marking algorithm is 1.5 -competitive. Analyzing the competitiveness of other non-uniform problems remains a challenging open problem.

Sleator and Tarjan [15] used a slightly different framework to study competitiveness in paging problems. They compared on-line algorithms to off-line algorithms with different numbers of servers (amounts of fast memory). They showed that LRU running with $k$ servers performs within a factor of $k /(k-h+1)$ of any off-line algorithm with $h \leq k$ servers and that this is the minimum competitive factor that can be achieved. Young [17] has extended this analysis to randomized algorithms. He has shown that the marking algorithm is roughly $2 \ln (k /(k-h+1))$-competitive under these circumstances.

There are many open problems involving the combining of on-line algorithms. Most notable of these is to extend the technique of constructing an algorithm competitive with several others to other problems besides the uniform server problem. Candidates include non-uniform server problems, maintaining a list [15], and snoopy caching [9]. 


\section{ACKNOWLEDGMENT}

The authors thank Jorge Stolfi and an anonymous referee for many helpful suggestions.

\section{REFERENCES}

1. P. Berman, H. Karloff, and G. Tardos. A competitive 3-server algorithm, in "First Annual ACM-SIAM Symposium on Discrete Algorithms, San Francisco, January 1990," pp. 280-290.

2. A. Borodin, M. Linial, and M. SaKs, An optimal online algorithm for metrical task systems, in "Proceedings 19th Annual ACM Symposium on Theory of Computing, New York, 1987," pp. 373-382.

3. A. Borodin, M. Linial, ANd M. SAKs, An optimal online algorithm for metrical task systems, $J . A C M$, to appear.

4. M. Chrobak, H. Karloff, T. Payne, and S. Vishwanathan, New results on server problems, in SLAM J. Discrete Math. 4, No. 2 (1991), 172-181.

5. M. Chrobak and L. LaRmore, "An optimal online algorithm for $k$ servers on trees," SLAM J. Comput. 20, (1991), 144-148.

6. D. Coppersmith, P. Doyle, P. Raghavan, and M. SNir, Random walks on weighted graphs and applications to on-line algorithms, in "Proceedings, 22nd Annual ACM Symposium on Theory of Computing, Baltimore, May 1990," pp. 369-378; Revised version available as an IBM research report.

7. $\Lambda$. Fiat, Y. Rabani, And Y. Ravid, Competitive $k$-server algorithms, in "Proceedings, 31st Annual Symposium on Foundations of Computer Science, St. Louis, 1990," pp. 454-463.

8. A. R. Karlin, M. S. Manasse, L. A. McGeoch, and S. Owicki, Competitive randomized algorithms for non-uniform problems, in "First Annual ACM-SIAM Symposium on Discrete Algorithms, San Francisco, January 1990," pp. 301-309.

9. A. R. Karlin, M. S. Manasse, L. Rudolph, and D. D. Sleator, Competitive snoopy caching, Algorithmica 3, No. 1 (1988), 79-119.

10. M. S. Manasse, L. A. McGeoch, and D. D. Sleator, Competitive algorithms for on-line problems, in "Proceedings, 20th Annual ACM Symposium on Theory of Computing, Chicago, 1988," pp. 322-333.

11. M. S. MANasse, L. A. McGeoch, and D. D. SLeator, Competitive algorithms for server problems, J. Algorithms 11, No. 2 (1990), 208-230.

12. L. A. McGeoch and D. D. Sleator, A strongly competitive randomized paging algorithm, Algorithmica, to appear.

13. P. Raghavan, Lecture notes on randomized algorithms, IBM research report, Yorktown Heights, 1990.

14. P. Raghavan and M. SNir, Memory versus randomization in on-line algorithms, in "Automata, Languages, and Programming," Lecture Notes in Computer Science, Vol. 372, pp. 687-703, Springer-Verlag, New York/Berlin, 1989; revised version available as an IBM research report.

15. D. D. Sleator and R. E. TARJan, Amortized efficiency of list update and paging rules, Comm. ACM 28, No. 2 (1985), 202-208.

16. A. C. YAO, Probabilistic computations: Towards a unified measure of complexity, in "Proceedings, 17th Annual Symposium on the Foundations of Computer Science, Chicago, November 1982," pp. 80-91.

17. N. Young, On-line caching as cache size varies, in "Second Annual ACM-SIAM Symposium on Discrete Algorithms, San Francisco, 1991," pp. 241-250. 